Article

\title{
Host and Cropping System Shape the Fusarium Population: 3ADON-Producers Are Ubiquitous in Wheat Whereas NIV-Producers Are More Prevalent in Rice
}

\author{
Meixin Yang ${ }^{1,+}{ }^{\dagger}$, Hao Zhang ${ }^{1,+}$, Xiangjiu Kong ${ }^{1}$, Theo van der Lee ${ }^{2}$, Cees Waalwijk ${ }^{2}$ (D), \\ Anne van Diepeningen ${ }^{2}$, Jin $X u^{1}$, Jingsheng $X u^{1}$, Wanquan Chen ${ }^{1, *}$ and Jie Feng ${ }^{1, *}$ \\ 1 State Key Laboratory for Biology of Plant Diseases and Insect Pests, Institute of Plant Protection, \\ Chinese Academy of Agriculture Sciences, Beijing 100193 China; yyangmeixin@126.com (M.Y.); \\ zhanghao@caas.cn (H.Z.); xjkong0528@126.com (X.K.); jinxu@ippcaas.cn (J.X.); jsxu@ippcaas.cn (J.X.) \\ 2 Wageningen Plant Research, P.O. Box 16, 6700 AA Wageningen, The Netherlands; \\ theo.vanderlee@wur.nl (T.v.d.L.); cees.waalwijk@wur.nl (C.W.); anne.vandiepeningen@wur.nl (A.v.D.) \\ * Correspondence: wqchen@ippcaas.cn (W.C.); jfeng@ippcaas.cn (J.F.); Tel.: +86-10-62815618 (W.C.); \\ +86-10-62815927 (J.F.) \\ + These authors contribute equally to this work.
}

Received: 5 February 2018; Accepted: 6 March 2018; Published: 8 March 2018

\begin{abstract}
In recent years, Fusarium head blight (FHB) outbreaks have occurred much more frequently in China. The reduction of burning of the preceding crop residues is suggested to contribute to more severe epidemics as it may increase the initial inoculum. In this study, a large number of Fusarium isolates was collected from blighted wheat spikes as well as from rice stubble with perithecia originating from nine sampling sites in five provinces in Southern China. Fusarium asiaticum dominated both wheat and rice populations, although rice populations showed a higher species diversity. Chemotype analysis showed that rice is the preferred niche for NIV mycotoxin producers that were shown to be less virulent on wheat. In contrast, 3ADON producers are more prevalent on wheat and in wheat producing areas. The $3 \mathrm{ADON}$ producers were shown to be more virulent on wheat, revealing the selection pressure of wheat on $3 \mathrm{ADON}$ producers. For the first time, members of the Incarnatum-clade of Fusarium Incarnatum-Equiseti Species Complex (FIESC) were found to reproduce sexually on rice stubble. The pathogenicity of FIESC isolates on wheat proved very low and this may cause the apparent absence of this species in the main wheat producing provinces. This is the first report of the Fusarium population structure including rice stubble as well as a direct comparison with the population on wheat heads in the same fields. Our results confirm that the perithecia on rice stubble are the primary inoculum of FHB on wheat and that cropping systems affect the local Fusarium population.
\end{abstract}

Keywords: cropping system; Fusarium head blight; chemotype; rice stubble

Key contribution: This study confirmed that the perithecia on rice stubble are the primary inoculum of FHB on wheat and that cropping systems affect the local Fusarium population.

\section{Introduction}

Fusarium head blight (FHB) is a devastating disease of wheat (Triticum aestivum L.), barley (Hordeum vulgare L.) and other small grains worldwide [1,2]. In these crops FHB causes reductions in yield and grain quality, and the harvested grains are often contaminated with mycotoxins, such as 
trichothecenes. Consumption of these grains and products might have pernicious effects on human and animal health [3].

Fusarium graminearum species complex (FGSC) consists of at least 16 phylogenetically distinct species [4]: F. graminearum is the most widely distributed species and occurs in most FHB areas around the world [5], while F. asiaticum is the main FHB pathogen present in Asia [2,6,7]. Members of FGSC produce different trichothecenes [8]: NIV and acetylated derivatives (NIV chemotype), DON and primarily 3-acetyldeoxynivalenol (3ADON chemotype), or DON and primarily 15 -acetyldeoxynivalenol (15ADON chemotype). These chemotypes may affect species or population ecology because the corresponding mycotoxins differ in toxicity and bioactivity [9]. A number of chemotype shifts were observed in different continents in recent years $[2,10,11]$. Moreover, in China, $3 \mathrm{ADON}$ producers were shown to have higher fitness than NIV producers as pathogens of FHB on wheat [2].

FHB pathogens usually overwinter on infested crop residues (corn stalks, rice and wheat straw, and other host plants). In spring, perithecia form on the surface of these residues, and ascospores released from perithecia on this crop debris serve as the primary inoculum of FHB. Hence, the presence of crop debris is essential for the overwintering of Fusarium. In agreement with this hypothesis, several studies have found a strong association between the distribution of FGSC species and cropping systems (host preference). F. asiaticum dominated in regions where rice is grown in rotation with wheat, as can be found in China and Korea, whereas F. graminearum was more common in wheat-maize rotation systems [2,12]. A similar situation was observed in the USA [13], while in Brazil, F. graminearum was found to be dominant in wheat [14], F. asiaticum in rice [15], and F. meridionale was determined as the predominant FGSC species on maize ears and stalks [16]. In South Africa, F. boothii was shown to be the best colonizer and mycotoxin producer on maize whereas $F$. graminearum was predominant on wheat $[17,18]$. Similar results were found in China, where F. boothii was prominent when maize is cultivated without rotation with wheat or rice [19]. However, nearly all studies in different agroecosystems focus on the pathogenic stage of Fusarium and there are few reports on the saprophytic stage on crops residues, which is crucial to the lifecycle and epidemiology of the pathogen. So far, there is no direct evidence for the overlap between Fusarium populations from wheat heads and the previous crop debris, which is important to understand the epidemiology of this pathogen.

In China, wheat-rice rotation within one year is the predominant cropping system in the regions along the Yangtze River, where FHB outbreaks are very frequent [2,20]. Abundant perithecia can be found on rice stubble in wheat fields in early April. Although they are usually thought to be the inoculum of FHB, the species and chemotype composition of this population are largely unknown. To study the role of the saprophytic phase of the lifecycle as a selective bottleneck, we collected a large number of Fusarium isolates from both wheat heads and rice stubble that were sampled from the same fields in five provinces along the Yangtze River. These populations were characterized at the species and chemotype level and their composition was compared. Subsequently, the pathogenicity of Fusarium species from wheat and from rice towards wheat was determined to identify possible differences in this host. Finally, these findings are discussed in the context of rice populations as the main primary inocula of FHB and the selection of wheat vs. rice on the different chemotypes of FGSC with respect to the different cropping systems.

\section{Results}

\subsection{Fusarium Species Determination}

A total of 1246 single spore strains were isolated from nine sampling sites in five provinces (Figure 1A), 702 from rice stubble and 544 from wheat kernels. Among them, 1186 isolates could be analyzed by the MLGT assay and they belonged to three species within FGSC: F. asiaticum $(n=1110)$, F. graminearum $(n=67)$, and F. meridionale $(n=9)$. Based on partial TEF- $1 \alpha$ gene sequences, the remaining 60 strains were identified as 10 species including F. incarnatum-F. equiseti Species Complexes (FIESC) 
$(n=30)$, F. acuminatum $(n=1)$, F. tricinctum $(n=1)$, F. armeniacum $(n=6)$, F. kyushuense $(n=2)$, F. fujikuroi $(n=7)$, F. proliferatum $(n=6)$, F. concentricum $(n=3)$, F. nygamai $(n=3)$, and F. verticillioides $(n=1)$. The distribution of these isolates is summarized in Table 1 . The species composition on wheat is simple, although eight species were identified, as $93.6 \%$ of the isolates belong to F. asiaticum. In the rice population, we found more species (12 species) than on wheat, but again F. asiaticum was predominant, although its frequency was lower $(85.6 \%)$. The fact that F. asiaticum dominated both populations indicates that it can circulate in the wheat-rice rotation system.

The majority of F. graminearum isolates 86.1\% (55/67) were isolated from Sichuan Province; F. graminearum is rarely found in the provinces located in the middle and lower reaches of the Yangtze River. All nine F. meridionale strains collected originated from Sichuan Province and exhibited a significantly (Fisher's Exact Test, $p<0.001$ ) uneven distribution among rice and wheat, as only one isolate originated from wheat and eight from rice. Most non-FGSC species are at very low frequency except FIESC, which accounted for 50\% of them (30/60). FIESC isolates were identified in all five provinces but were most commonly found on rice stubble $(27 / 30)$. Phylogenetic analysis of FIESC showed that almost all isolates (29/30) belong to the Incarnatum clade with the exception of one isolate (IPP 14080) that belonged to the Equiseti clade (Figure 2).

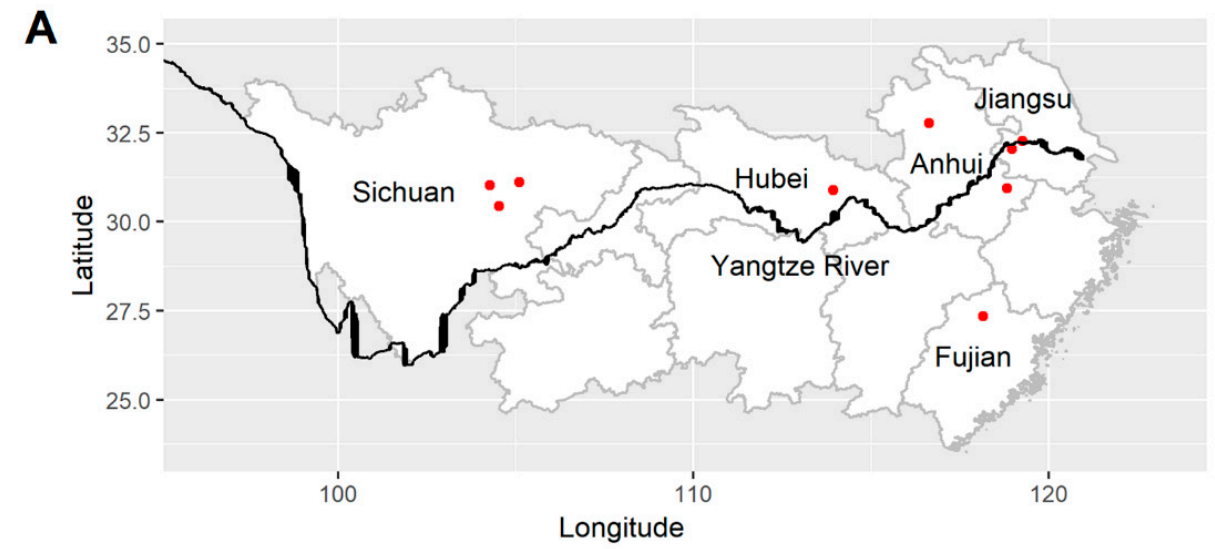

B

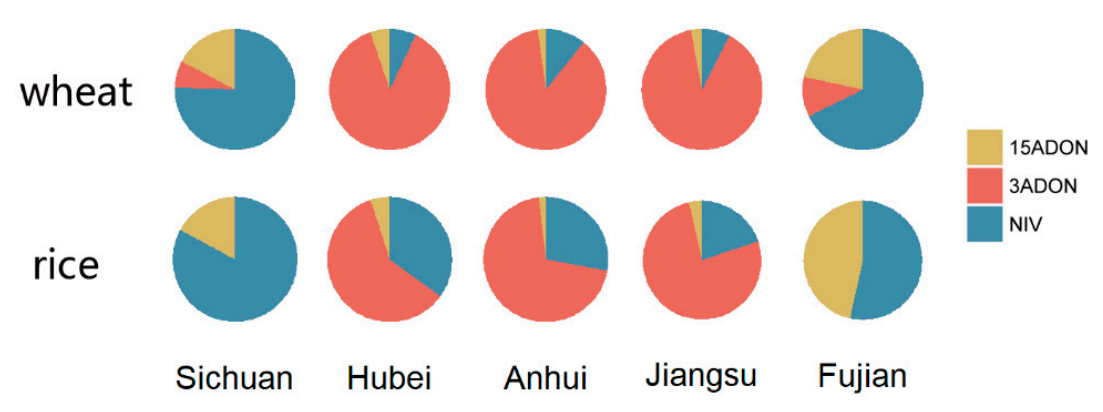

Figure 1. (A) Map of Southern China indicating the nine sampling sites in five provinces. (B) Trichothecenes chemotypes composition of wheat and rice populations in each province. 


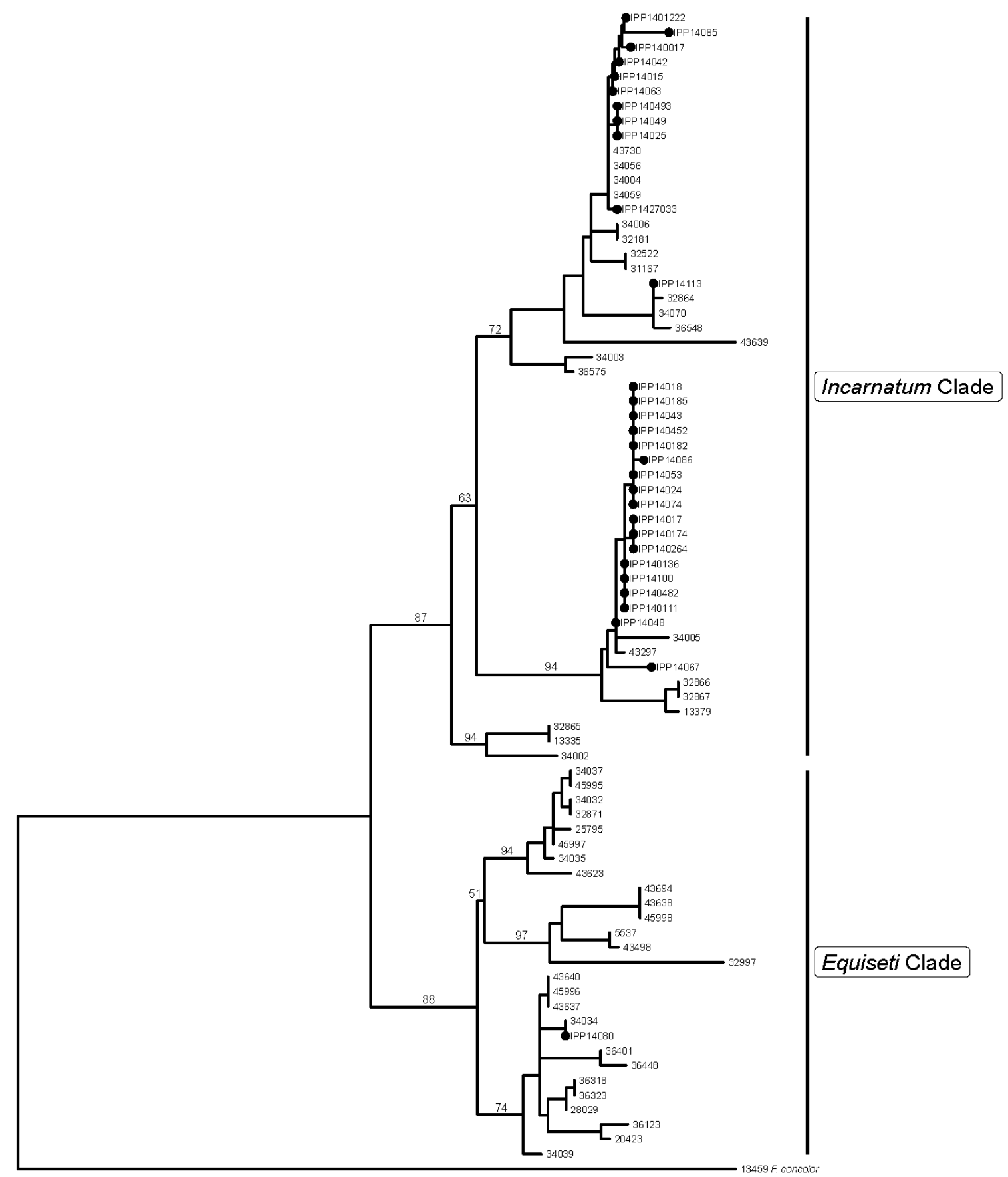

Figure 2. Maximum likelihood tree inferred from TEF-1 $\alpha$ sequences by PhyML in Geneious 9.1. Strains labeled by a black dot were sequenced in this study, others are reference strains from O'Donnell et al., 2009. F. concolor (NRRL 13459) was set as an outgroup. Numbers above the branches are bootstrap values. 
Table 1. Distribution of Fusarium isolates from wheat and rice in Southern China.

\begin{tabular}{|c|c|c|c|c|c|c|c|c|c|c|c|c|c|c|c|c|c|c|c|c|}
\hline \multirow{3}{*}{ Species Complex * } & \multirow{3}{*}{$\begin{array}{c}\text { Provinces } \\
\text { Sampling Sites } \\
\text { Hosts } * *\end{array}$} & \multicolumn{6}{|c|}{ Sichuan } & \multirow{2}{*}{\multicolumn{2}{|c|}{$\begin{array}{c}\text { Hubei } \\
\text { Xiaogan }\end{array}$}} & \multicolumn{4}{|c|}{ Anhui } & \multicolumn{4}{|c|}{ Jiangsu } & \multirow{2}{*}{\multicolumn{2}{|c|}{$\begin{array}{c}\text { Fujian } \\
\text { Nanping }\end{array}$}} & \multirow{3}{*}{ Total } \\
\hline & & \multicolumn{2}{|c|}{ Jianyang } & \multicolumn{2}{|c|}{ Guanghan } & \multicolumn{2}{|c|}{ Mianyang } & & & \multicolumn{2}{|c|}{ Fengtai } & \multicolumn{2}{|c|}{ Xuanzhou } & \multicolumn{2}{|c|}{ Nanjing } & \multicolumn{2}{|c|}{ Yizheng } & & & \\
\hline & & W & $\mathbf{R}$ & $\mathbf{W}$ & $\mathbf{R}$ & $\mathbf{W}$ & $\mathbf{R}$ & $\mathbf{W}$ & $\mathbf{R}$ & $\mathbf{W}$ & $\mathbf{R}$ & $\mathbf{W}$ & $\mathbf{R}$ & $\mathbf{W}$ & $\mathbf{R}$ & $\mathbf{W}$ & $\mathbf{R}$ & $\mathbf{W}$ & $\mathbf{R}$ & \\
\hline \multirow{3}{*}{ FGSC } & F. asiaticum & 17 & 56 & 38 & 49 & 28 & 74 & 57 & 60 & 42 & 28 & 50 & 81 & 28 & 75 & 37 & 65 & 211 & 114 & 1110 \\
\hline & F. graminearum & 4 & 21 & 1 & 1 & 14 & 14 & 0 & 0 & 1 & 1 & 0 & 1 & 0 & 1 & 2 & 0 & 6 & 0 & 67 \\
\hline & F. meridionale & 1 & 5 & 0 & 1 & 0 & 2 & 0 & 0 & 0 & 0 & 0 & 0 & 0 & 0 & 0 & 0 & 0 & 0 & 9 \\
\hline FIESC & & 0 & 0 & 0 & 1 & 0 & 0 & 0 & 21 & 0 & 0 & 0 & 2 & 0 & 0 & 0 & 1 & 3 & 2 & 30 \\
\hline \multirow[b]{2}{*}{ FTSC } & F. acuminatum & 0 & 0 & 0 & 0 & 0 & 0 & 0 & 1 & 0 & 0 & 0 & 0 & 0 & 0 & 0 & 0 & 0 & 0 & 1 \\
\hline & F. tricinctum & 0 & 0 & 0 & 0 & 0 & 0 & 0 & 1 & 0 & 0 & 0 & 0 & 0 & 0 & 0 & 0 & 0 & 0 & 1 \\
\hline FSPSC & F. armeniacum & 0 & 0 & 0 & 0 & 0 & 0 & 0 & 0 & 0 & 0 & 0 & 6 & 0 & 0 & 0 & 0 & 0 & 0 & 6 \\
\hline FSAMSC & F. kyushuense & 0 & 0 & 0 & 0 & 0 & 0 & 0 & 1 & 0 & 0 & 0 & 0 & 0 & 0 & 0 & 0 & 1 & 0 & 2 \\
\hline \multirow{6}{*}{ FFSC } & F. fujikuroi & 0 & 0 & 0 & 0 & 0 & 0 & 0 & 0 & 0 & 0 & 0 & 0 & 1 & 0 & 0 & 6 & 0 & 0 & 7 \\
\hline & F. proliferatum & 0 & 2 & 0 & 0 & 0 & 0 & 0 & 0 & 0 & 0 & 0 & 0 & 0 & 0 & 0 & 4 & 0 & 0 & 6 \\
\hline & F. concentricum & 0 & 3 & 0 & 0 & 0 & 0 & 0 & 0 & 0 & 0 & 0 & 0 & 0 & 0 & 0 & 0 & 0 & 0 & 3 \\
\hline & F. nygamai & 0 & 0 & 0 & 0 & 1 & 0 & 0 & 0 & 0 & 0 & 0 & 0 & 0 & 2 & 0 & 0 & 0 & 0 & 3 \\
\hline & F. verticillioides & 0 & 0 & 0 & 0 & 0 & 0 & 0 & 0 & 0 & 0 & 1 & 0 & 0 & 0 & 0 & 0 & 0 & 0 & 1 \\
\hline & Total & 22 & 87 & 39 & 52 & 43 & 90 & 57 & 84 & 43 & 29 & 51 & 90 & 29 & 78 & 39 & 76 & 221 & 116 & 1246 \\
\hline
\end{tabular}

* Species complexes: FGSC, F. graminearum species complex; FIESC, F. incarnatum-equiseti species complex; FTSC, F. tricinctum species complex; FSPSC, F. sporotrichioides species complex;

FSAMSC, F. sambucinum species complex; and FFSC, F. fujikuroi species complex. ${ }^{*} \mathrm{~W}$ represents wheat and $\mathrm{R}$ represents rice. 


\subsection{Trichothecene Chemotype Identification}

Trichothecene chemotypes of all 1186 FGSC isolates were identified by MLGT. The results are shown in Table 2. All F. graminearum and F. meridionale isolates are 15ADON and NIV producers, respectively. In the predominant species, F. asiaticum, all three chemotypes were found. Strains producing NIV (96.6\%) dominated in Sichuan Province, especially on rice, where no other chemotypes were found. $3 \mathrm{ADON}$ is the main chemotype (80.5\%) in the Hubei, Anhui, and Jiangsu Provinces, which are located in the middle and lower reaches of the Yangtze River. However, we found an uneven distribution of chemotypes in rice and wheat populations in these provinces. Significantly higher ratios (Fisher's Exact Test, $p<0.001)$ of NIV producers were observed on rice stubble (20-35\%) than on wheat kernels (7-10\%) in these provinces (Figure 1B). In the nursery in Fujian Province, NIV was the main chemotype with the ratio of $64.0 \%$. It is interesting that in Fujian Province up to $28.9 \%$ of F. asiaticum isolates were $15 \mathrm{ADON}$ producers, which was rare in other provinces we tested. $3 \mathrm{ADON}$-producing F. asiaticum were only found in the wheat population with a low frequency $(7.1 \%)$.

Table 2. Trichothecene type compositions of FGSC isolates.

\begin{tabular}{|c|c|c|c|c|c|c|c|}
\hline \multirow{2}{*}{ Hosts } & \multirow{2}{*}{ Provinces } & \multicolumn{3}{|c|}{ F. asiaticum } & \multirow{2}{*}{$\begin{array}{c}\text { F. graminearum } \\
\text { 15ADON }\end{array}$} & \multirow{2}{*}{$\frac{\text { F. meridionale }}{\text { NIV }}$} & \multirow{2}{*}{ Total } \\
\hline & & NIV & 3ADON & 15ADON & & & \\
\hline \multirow{5}{*}{ Wheat } & Sichuan & 74 & 8 & 1 & 19 & 1 & 103 \\
\hline & Hubei & 4 & 50 & 3 & 0 & 0 & 57 \\
\hline & Anhui & 10 & 81 & 1 & 1 & 0 & 93 \\
\hline & Jiangsu & 5 & 60 & 0 & 2 & 0 & 67 \\
\hline & Fujian & 147 & 23 & 41 & 6 & 0 & 217 \\
\hline \multirow{6}{*}{ Rice } & Sichuan & 179 & 0 & 0 & 36 & 8 & 223 \\
\hline & Hubei & 21 & 36 & 3 & 0 & 0 & 60 \\
\hline & Anhui & 31 & 78 & 0 & 2 & 0 & 111 \\
\hline & Jiangsu & 28 & 108 & 4 & 1 & 0 & 141 \\
\hline & Fujian & 61 & 0 & 53 & 0 & 0 & 114 \\
\hline & Total & 560 & 444 & 106 & 67 & 9 & 1186 \\
\hline
\end{tabular}

\subsection{Pathogenicity Analyses}

In order to understand how the hosts (wheat or rice) could influence their distribution of Fusarium species chemotype combinations, we selected 10 strains of each population including F. graminearum, F. meridionale, and FIESC as well as NIV, 3ADON, or 15ADON producers of F. asiaticum for pathogenicity analyses. Ten strains were selected randomly from each population. The three populations of F. graminearum (15ADON), F. asiaticum (3ADON), and F. asiaticum (15ADON) showed the highest incidence of infected spikelets (IIS), at $41.5 \pm 5.2,43.3 \pm 6.3$, and $45.1 \pm 4.2$, respectively. NIV-type F. asiaticum strains were clearly less aggressive ( $27.5 \pm 3.1)$, but their pathogenicity was still significantly higher than that of F. meridionale isolates (22.6 \pm 3.0$)$, while FIESC strains showed the lowest IIS $(6.3 \pm 1.0)$, as most isolates in this population only caused symptoms in the inoculated spikelet which never extended to adjacent spikelets (Figure 3). 
60

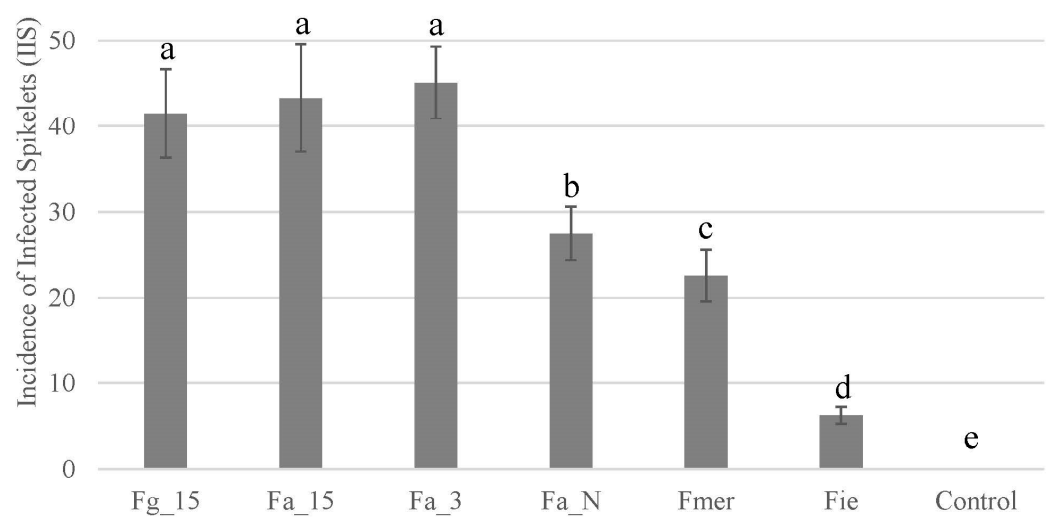

Figure 3. Pathogenicity of different Fusarium populations on wheat. Fg_15: 15ADON-type F. graminearum; Fa_15: 15ADON-type F. asiaticum; Fa_3: 3ADON-type F. asiaticum; Fa_N: NIV-type F. asiaticum; Fmer: F. meridionale; Fie: FIESC.

\section{Discussion}

FHB is one of the most important problems in wheat production worldwide. In the last decade, FHB outbreaks became much more frequent in China, where the epidemic area covered over 4 million ha in seven of 10 years. The popularization of straw-returning instead of burning of the preceding crops was suggested as the main reason, as it leads to increased numbers of pathogen propagules in the field. In this study, we surveyed the Fusarium composition on wheat kernels and on rice stubble in the same locations in five provinces of Southern China. The rice populations revealed higher species diversity than the wheat populations. F. asiaticum dominated both pathogenic and saprophytic populations, and there is no significant difference ( $p=0.29$, Fisher's Exact Test) between the ratio of F. asiaticum in the two populations. This indicated that rice stubble may be selective for F. asiaticum rather than for other FHB species. It also confirmed that the perithecia on rice stubble are likely to act as the primary inoculum for FHB of wheat in Southern China. Del Ponte et al. (2015) compared the Fusarium composition on wheat heads and on corn residue in Brazil and observed a significant difference in two places; while F. meridionale dominated the saprophytic stage, F. graminearum was predominantly found in the pathogenic stage [14]. This may be due to the lower pathogenicity of F. meridionale on wheat-although they may show better fitness on corn residue (saprophytic stage), they failed in the competition with F. graminearum on wheat (pathogenic stage). In this study, we found similar pathogenicity levels in F. asiaticum with the 3ADON genotype and F. graminearum with the $15 \mathrm{ADON}$ genotype. We thus hypothesize that the preference of rice stubble in the saprophytic stage is crucial. Most F. graminearum strains (55/67) were isolated from Sichuan Province, which is consistent with our previous report [2]. In this region, although rice is prevalent, maize cultivation is also popular, which might explain the higher frequency of F. graminearum as F. graminearum is prevalent on maize.

In agreement with previous studies in China [2,21-23], we found F. asiaticum with the 3ADON genotype to dominate the Middle-Lower Yangtze River Plain, which is the area most frequently affected by FHB epidemics. However, NIV-producing F. asiaticum isolates were reported to be associated with almost all rice agroecosystems outside China. This difference may be caused by the selection on wheat. A strict wheat-rice rotation within one year is unique to this large area in China. Consequently, the Fusarium population is forced to go through two selection cycles, one on rice and one on wheat every year. In agreement with previous studies [2,24], we found that the pathogenicity of $F$. asiaticum with $3 \mathrm{ADON}$ and F. graminearum with $15 \mathrm{ADON}$ was significant higher than that of $F$. asiaticum with the NIV genotype on wheat (Figure 3). The better fitness of $F$. asiaticum on rice and DON producers on wheat leads to the prevalence of $3 \mathrm{ADON}$-producing $F$. asiaticum in most wheat-rice rotation areas in Southern China. The observed chemotype composition supports this conclusion, as the ratio of 3ADON 
producers in wheat is significantly higher ( $p<0.001$, Fisher's Exact Test) than that on rice in Hubei, Anhui, and Jiangsu, while in Sichuan and Fujian, 3ADON producers were only isolated on wheat but not on rice (Figure 1B, Table 2). In the absence of selection pressure by wheat, NIV-producing F. asiaticum dominates the population on rice seed in rice growing areas in South Korea [12] and Brazil [15]. Small populations of F. asiaticum with the NIV genotype were found on wheat that was grown near rice-growing areas in United States [13] and Brazil [14], and the inoculum was speculated to come from adjacent rice fields, possibly causing less frequent infections because of their lower virulence compared with DON-producing F. graminearum. In Eastern Uruguay, NIV-type F. asiaticum and 15ADON-type F. graminearum showed similar ratios (52\% vs. $43 \%$ ) in Cerro Largo, which is a new wheat production zone in traditionally rice growing areas. This can be explained by assuming that NIV-producing F. asiaticum isolates represent an old FHB population that existed before wheat was implemented, while the replacement by $15 \mathrm{ADON}$-producing $F$. graminearum is still in progress in the new wheat production zone. Monitoring the species and chemotype shift of FGSC in this region could further validate our hypothesis and may be important for food safety because of the differences in toxicity of the trichothecenes.

Different from the provinces in the Middle-Lower Yangtze River Plain, the less virulent NIV population dominated both wheat and rice populations in Sichuan Province, which is traditionally a wheat-rice rotation region. In Sichuan Province isolates with the 3ADON chemotype were only isolated on wheat and at low frequency $(9.5 \%)$. This may be due to the decrease of the wheat production in Sichuan. The mountain region in this province restricts the use of machines and, along with the increase of the labor costs, more and more farmers have abandoned wheat cultivation. Consequently, the selection pressure for more virulent species on wheat also decreased. In addition, the mountains and the small holder farming in this region also limits the spread of successful populations on wheat. In Japan, where wheat cultivation is rare and rice production occurs across the entire country, $70 \%$ of the F. asiaticum isolates on wheat are NIV-type, much higher than 3ADON producers (29\%) [6]. Also, in this case the lack of selection on wheat could be responsible for the prevalence of the F. asiaticum NIV producers. In Fujian, the situation is distinct from other provinces in this study because farmers abandoned wheat cultivation due to severe FHB epidemics more than 10 years ago. In the present study, we could only collect samples in a small nursery dedicated to evaluating FHB resistance of wheat varieties from other provinces. NIV-producing F. asiaticum dominated both wheat and rice populations and only a few $3 A D O N$ producers were found on wheat, which may be introduced by wheat seeds from the Middle-Lower Yangtze River Plain. The presence of abundant NIV-type F. asiaticum in non- (Fujian) and less-wheat (Sichuan) provinces revealed that not only wheat selects $3 \mathrm{ADON}$ producers, but also that rice agroecosystems favor NIV producers. This strong association between NIV producers and rice is in agreement with previous reports from Korea [12], Japan [6], the United States [13], Uruguay [11], and Brazil [15], further underpinning this conclusion. Additional evidence was found in the Middle-Lower Yangtze River Plain, where in all three provinces significantly higher ratios of NIV producers were observed on rice stubble (20-35\%) than on wheat kernels $(7-10 \%)$, despite the fact that $3 \mathrm{ADON}$ is the main chemotype in this region (Figure 1B, Table 2). In agreement with previous reports [23,24], we also found a high ratio of $15 \mathrm{ADON}$-producing F. asiaticum on both rice and on wheat in Fujian, which is rare in wheat growing areas. This population showed a similar level of pathogenicity to $3 \mathrm{ADON}$-producing F. asiaticum and 15ADON-producing F. graminearum on wheat, but higher aggressiveness than NIV producers of F. asiaticum and F. meridionale (Figure 3).

All nine F. meridionale isolates collected originated from Sichuan. Previous studies reported the presence of F. meridionale in Sichuan and Yunnan on wheat [2] and Sichuan and southern Gansu on maize [19] in China. Similar to our study, it was typically found in low frequencies. Based on the reports so far, it seems that this species is limited to southwest China. F. meridionale has been reported as the main species on maize in Nepal and in Northern Argentina $[25,26]$. Small populations of $F$. meridionale were also found in Korea [7] and in South Africa [18]. Recently, a large F. meridionale population was identified on wheat in Brazil, which was thought to be related to the two harvests of maize in 
one year [14]. F. meridionale was also shown to be less competitive on wheat than F. graminearum isolates [14]. Similar results were obtained in this study, as we found F. meridionale to be less virulent than both $F$. graminearum and F. asiaticum on wheat. This may be the main reason for the uneven distribution of $F$. meridionale on wheat (1/9) vs. rice stubble (8/9).

In addition to FGSC, 60 non-FGSC Fusarium isolates where obtained and half of these strains were identified as FIESC. Among them, three isolates were found on wheat while the other 27 were isolated from rice stubble. In the pathogenicity assays, most FIESC isolates caused only symptoms on the inoculated spikelets but no spreading was observed, which may explain the absence of this species in the main wheat producing regions. Phylogenetic analysis showed that 29 strains were clustered in the Incarnatum clade and the remaining one in the Equiseti clade. Lineages in the Incarnatum clade are not known to have a sexual stage [27]. However, the rice stubble population in our study was obtained as single spores isolated from ascospores (29 strains from five provinces). Therefore, we hypothesize that a natural sexual cycle occurs on rice and it may be important to study this sexual cycle and its consequences for population diversity and epidemiology in more detail.

Many previous studies on the occurrence and prevalence of FGSC exist and the composition of populations is reported to change over time [28]. However, the drivers for this phenomenon are still largely unknown. Recently, based on available data from the literature, we concluded that a cropping system with wheat/maize rotation selects for F. graminearum, while a wheat/rice rotation selects for F. asiaticum. F. boothii is selected when maize is cultivated without rotation [19]. In this study, we further investigated the Fusarium population on rice stubble on a large scale and made comparisons with populations from wheat kernels. We confirmed that perithecia on rice stubble were the primary inoculum for FHB on wheat. Based on our findings and previous reports across China, we conclude that wheat is selective for DON-producing F. graminearum and F. asiaticum due to higher pathogenic fitness. In contrast, rice favors F. asiaticum with the NIV chemotype, possibly due to increased saprophytic fitness. Depending on the cropping system, this divergent selection can shape Fusarium populations and this can help us to predict possible prevalent species and subsequently predict which mycotoxin contamination can be expected.

\section{Materials and Methods}

\subsection{Fungal Isolates}

Rice stubble with black perithecia was collected from nine sampling sites in five provinces (Figure 1A) in Southern China from 30 March to 7 April 2014. Sichuan, Hubei, Anhui, and Jiangsu Provinces are common wheat producing areas and wheat-rice rotation is the most prevalent cropping system. However, there is no wheat grown in Fujian Province, and the sampling site Nanping is just a small nursery where wheat is rotated with rice. Sichuan Province is a mountainous area, located in the upper valley of the Yangtze River. Hubei, Anhui, and Jiangsu Provinces are located in the Middle-Lower Yangtze River Plain. Diseased wheat spikes with mature kernels were sampled in the same place from 27 April to 10 May 2014. For wheat samples, diseased kernels were surface-sterilized in 70\% ethanol for $30 \mathrm{~s}$, and immediately immersed in $2 \%$ sodium hypochlorite for $90 \mathrm{~s}$, after which the kernels were extensively rinsed with sterile distilled water and placed on potato dextrose agar (PDA) plates. After 3 days of incubation at $26^{\circ} \mathrm{C}$, newly grown-out mycelium was transferred into flasks containing $20 \mathrm{~mL}$ of mung bean broth cultures ( $3 \%$ mung bean extract). Flasks were shaken at $180 \mathrm{rpm}$ for 2 days at $26^{\circ} \mathrm{C}$. Spore suspensions were diluted to 100 -fold and plated on $1 \%$ water agar. The plates were incubated for 12 to $24 \mathrm{~h}$ and single spores were identified and transferred to PDA plates. Single spore cultures were stored in $15 \%$ dimethyl sulfoxide (DMSO) at $-80{ }^{\circ} \mathrm{C}$. For rice samples, the rice stubble with perithecia were excised and surface-sterilized as described above. Small pieces of perithecia were picked up and soaked in $100 \mu \mathrm{L}$ sterile distilled water in PCR tubes. After 6-8 h incubation at room temperature, ascospores were allowed to be released in the water. Subsequently, single spore isolations were performed as described above. Strain information is provided in Table S1. 


\subsection{Genomic DNA Extraction}

A small mycelial plug was transferred to 6-cm Petri plates containing potato dextrose agar (PDA) and incubated at $26^{\circ} \mathrm{C}$ for 5 days. Mycelium was harvested with scalpel blades and vacuum freeze-dried at $-20^{\circ} \mathrm{C}$ overnight. The mycelium was lyophilized using liquid nitrogen and grinding by vigorous shaking in a MiniBeadbeater-96 (Biospec, Bartlesville, OK, USA). Total genomic DNA was extracted using the SP Fungal DNA Kit (Omega Biotek, Norcross, GA, USA) according to the manufacturer's instructions. DNA concentration was determined by NanoVue Plus (GE, Pittsburgh, PA, USA). Finally, DNA samples were diluted to $5 \mathrm{ng} / \mathrm{mL}$ for each sample in $96-$ well microtiter plates, which were stored at $-20^{\circ} \mathrm{C}$.

\subsection{Species and Trichothecene Chemotype Determination}

Multilocus genotyping (MLGT) assay [10] was used to determine the species and trichothecene chemotype of all strains. For the isolates that could not be identified by MLGT, partial translation elongation factor (TEF-1 $\alpha, 700 \mathrm{bp}$ ) gene sequences were generated with the previously reported primers [29]. These strains were identified by sequence comparison in the Fusarium MLST database [30] and/or in GenBank. Based on the TEF-1 $\alpha$ sequence, a maximum likelihood tree of FIESC isolates together with several reference strains (from O'Donnell et al., 2009) was constructed in Geneious 9.1 using PhyML, and F. concolor (NRRL 13459) was set as an outgroup.

\subsection{Pathogenicity Analyses}

A medium resistant winter wheat cultivar, Yangmai 158, was used for the pathogenicity tests. Wheat was planted in three different blocks $(2 \mathrm{~m} \times 4 \mathrm{~m})$ according to normal agronomic practices at the Langfang Farm (Experiment Farm of the Institute of Plant Protection) in 2015. We selected 10 strains from each population randomly (nine isolates from $F$. meridionale population) for pathogenicity analyses. Conidia were prepared by mung bean broth cultures as mentioned above. At anthesis, 10 heads in each block were inoculated by injecting $20 \mathrm{~mL}$ conidial suspension $\left(10^{6}\right.$ conidia $\left./ \mathrm{mL}\right)$ of one isolate into a central floret of a spike (injection inoculation). A total of 30 heads was infected by a single strain. Ten heads in each block were also selected to be inoculated with $20 \mu \mathrm{L}$ sterilized water as a control group. Pathogenicity was assessed as the incidence of infected spikelets, which was determined visually by counting the number of infected spikelets per head at 14 days after inoculation and expressed as a percentage of the total number of spikelets.

Supplementary Materials: The following are available online at www.mdpi.com/2072-6651/10/3/115/s1, Table S1: Strains information in this study.

Acknowledgments: This project received funding from the National Key R\&D Program of China (2016YFE0112900, 2016YFD0300705) and the European Union's Horizon 2020 research and innovation programme under grant agreement No. 678781 (MycoKey) and the National Natural Science Foundation of China (No. 31201477) and Fundamental Research Funds for Central Non-Profit Scientific Institution (Y2017XM01).

Author Contributions: H.Z., W.C. and J.F. conceived and designed the experiments; M.Y. and X.K. performed the experiments; M.Y. and H.Z. analyzed the data; J.X. (Jin Xu) and J.X. (Jingsheng Xu) contributed experimental techniques; M.Y., H.Z., A.v.D., T.v.d.L. and C.W. wrote the paper.

Conflicts of Interest: The authors declare no conflict of interest. The founding sponsors had no role in the design of the study; in the collection, analyses, or interpretation of data; in the writing of the manuscript, and in the decision to publish the results.

\section{References}

1. Kelly, A.C.; Clear, R.M.; O’Donnell, K.; McCormick, S.; Turkington, T.K.; Tekauz, A.; Gilbert, J.; Kistler, H.C.; Busman, M.; Ward, T.J. Diversity of Fusarium head blight populations and trichothecene toxin types reveals regional differences in pathogen composition and temporal dynamics. Fungal Genet. Biol. 2015, 82, 22-31. [CrossRef] [PubMed] 
2. Zhang, H.; Van der Lee, T.; Waalwijk, C.; Chen, W.Q.; Xu, J.; Xu, J.S.; Zhang, Y.; Feng, J. Population analysis of the Fusarium graminearum species complex from wheat in China show a shift to more aggressive isolates. PLoS ONE 2012, 7, e31722. [CrossRef] [PubMed]

3. Goswami, R.S.; Kistler, H.C. Heading for disaster: Fusarium graminearum on cereal crops. Mol. Plant Pathol. 2004, 5, 515-525. [CrossRef] [PubMed]

4. Sarver, B.A.; Ward, T.J.; Gale, L.R.; Broz, K.; Corby Kistler, H.; Aoki, T.; Nicholson, P.; Carter, J.; O’Donnell, K. Novel Fusarium head blight pathogens from Nepal and Louisiana revealed by multilocus genealogical concordance. Fungal Genet. Biol. 2011, 48, 1096-1107. [CrossRef] [PubMed]

5. O’Donnell, K.; Ward, T.J.; Aberra, D.; Kistler, H.C.; Aoki, T.; Orwig, N.; Kimura, M.; Bjornstad, S.; Klemsdal, S.S. Multilocus genotyping and molecular phylogenetics resolve a novel head blight pathogen within the Fusarium graminearum species complex from Ethiopia. Fungal Genet. Biol. 2008, 45, 1514-1522. [CrossRef] [PubMed]

6. Suga, H.; Karugia, G.W.; Ward, T.; Gale, L.R.; Tomimura, K.; Nakajima, T.; Miyasaka, A.; Koizumi, S.; Kageyama, K.; Hyakumachi, M. Molecular characterization of the Fusarium graminearum species complex in Japan. Phytopathology 2008, 98, 159-166. [CrossRef] [PubMed]

7. Lee, J.; Kim, H.; Jeon, J.J.; Kim, H.S.; Zeller, K.A.; Carter, L.L.; Leslie, J.F.; Lee, Y.W. Population structure of and mycotoxin production by Fusarium graminearum from maize in South Korea. Appl. Environ. Microbiol. 2012, 78, 2161-2167. [CrossRef] [PubMed]

8. Miller, J.D.; Greenhalgh, R.; Wang, Y.Z.; Lu, M. Trichothecene chemotypes of three Fusarium species. Mycologia 1991, 83, 121-130. [CrossRef]

9. Kimura, M.; Kaneko, I.; Komiyama, M.; Takatsuki, A.; Koshino, H.; Yoneyama, K.; Yamaguchi, I. Trichothecene 3-O-acetyltransferase protects both the producing organism and transformed yeast from related mycotoxins. Cloning and characterization of Tri101. J. Biol. Chem. 1998, 273, 1654-1661. [CrossRef] [PubMed]

10. Ward, T.J.; Clear, R.M.; Rooney, A.P.; O’Donnell, K.; Gaba, D.; Patrick, S.; Starkey, D.E.; Gilbert, J.; Geiser, D.M.; Nowicki, T.W. An adaptive evolutionary shift in Fusarium head blight pathogen populations is driving the rapid spread of more toxigenic Fusarium graminearum in North America. Fungal Genet. Biol. 2008, 45, 473-484. [CrossRef] [PubMed]

11. Umpierrez-Failache, M.; Garmendia, G.; Pereyra, S.; Rodriguez-Haralambides, A.; Ward, T.J.; Vero, S. Regional differences in species composition and toxigenic potential among Fusarium head blight isolates from Uruguay indicate a risk of nivalenol contamination in new wheat production areas. Int. J. Food Microbiol. 2013, 166, 135-140. [CrossRef] [PubMed]

12. Lee, J.; Chang, I.Y.; Kim, H.; Yun, S.H.; Leslie, J.F.; Lee, Y.W. Genetic diversity and fitness of Fusarium graminearum populations from rice in Korea. Appl. Environ. Microbiol. 2009, 75, 3289-3295. [CrossRef] [PubMed]

13. Gale, L.R.; Harrison, S.A.; Ward, T.J.; O’Donnell, K.; Milus, E.A.; Gale, S.W.; Kistler, H.C. Nivalenol-type populations of Fusarium graminearum and F. asiaticum are prevalent on wheat in southern Louisiana. Phytopathology 2011, 101, 124-134. [CrossRef] [PubMed]

14. Del Ponte, E.M.; Spolti, P.; Ward, T.J.; Gomes, L.B.; Nicolli, C.P.; Kuhnem, P.R.; Silva, C.N.; Tessmann, D.J. Regional and field-specific factors affect the composition of Fusarium head blight pathogens in subtropicalno-till wheat agroecosystem of Brazil. Phytopathology 2015, 105, 246-254. [CrossRef] [PubMed]

15. Gomes, L.B.; Ward, T.J.; Badiale-Furlong, E.; Del Ponte, E.M. Species composition, toxigenic potential and pathogenicity of Fusarium graminearum species complex isolates from southern Brazilian rice. Plant Pathol. 2015, 64, 980-987. [CrossRef]

16. Kuhnem, P.R.; Ward, T.J.; Silva, C.N.; Spolti, P.; Ciliato, M.L.; Tessmann, D.J.; Del Ponte, E.M. Composition and toxigenic potential of the Fusarium graminearum species complex from maize ears, stalks and stubble in Brazil. Plant Pathol. 2016, 65, 1185-1191. [CrossRef]

17. Beukes, I.; Rose, L.J.; van Coller, G.J.; Viljoen, A. Disease development and mycotoxin production by the Fusarium graminearum species complex associated with South African maize and wheat. Eur. J. Plant Pathol. 2018, 150, 893-910. [CrossRef]

18. Boutigny, A.-L.; Ward, T.J.; Coller, G.J.V.; Flett, B.; Lamprecht, S.C.; O’Donnell, K.; Viljoen, A. Analysis of the Fusarium graminearum species complex from wheat, barley and maize in South Africa provides evidence of species-specific differences in host preference. Fungal Genet. Biol. 2011, 48, 914-920. [CrossRef] [PubMed] 
19. Zhang, H.; Brankovics, B.; Luo, W.; Xu, J.; Xu, J.S.; Guo, C.; Guo, J.G.; Jin, S.L.; Chen, W.Q.; Feng, J. Crops are a main driver for species diversity and the toxigenic potential of Fusarium isolates in maize ears in China. World Mycotoxin J. 2016, 9, 701-715. [CrossRef]

20. Zhang, J.B.; Wang, J.H.; Gong, A.D.; Chen, F.F.; Song, B.; Li, X.; Li, H.P.; Peng, C.H.; Liao, Y.C. Natural occurrence of fusarium head blight, mycotoxins and mycotoxin-producing isolates of Fusarium in commercial fields of wheat in Hubei. Plant Pathol. 2013, 62, 92-102. [CrossRef]

21. Zhang, J.B.; Li, H.P.; Dang, F.J.; Qu, B.; Xu, Y.B.; Zhao, C.S.; Liao, Y.C. Determination of the trichothecene mycotoxin chemotypes and associated geographical distribution and phylogenetic species of the Fusarium graminearum clade from China. Mycol. Res. 2007, 111, 967-975. [CrossRef] [PubMed]

22. Qiu, J.B.; Xu, J.H.; Shi, J.R. Molecular characterization of the Fusarium graminearum species complex in Eastern China. Eur. J. Plant Pathol. 2014, 139, 811-823. [CrossRef]

23. Zhang, H.; Zhang, Z.; van der Lee, T.; Chen, W.Q.; Xu, J.; Xu, J.S.; Yang, L.; Yu, D.; Waalwijk, C.; Feng, J. Population genetic analyses of Fusarium asiaticum populations from barley suggest a recent shift favoring 3ADON producers in southern China. Phytopathology 2010, 100, 328-336. [CrossRef] [PubMed]

24. Puri, K.D.; Saucedo, E.S.; Zhong, S. Molecular characterization of Fusarium head blight pathogens sampled from a naturally infected disease nursery used for wheat breeding programs in China. Plant Dis. 2012, 96, 1280-1285. [CrossRef]

25. Desjardins, A.E.; Proctor, R.H. Genetic diversity and trichothecene chemotypes of the Fusarium graminearum clade isolated from maize in Nepal and identification of a putative new lineage. Fungal Genet. Biol. 2011, 115, 38-48. [CrossRef] [PubMed]

26. Sampietro, D.A.; Ficoseco, M.E.; Jimenez, C.M.; Vattuone, M.A.; Catalan, C.A. Trichothecene genotypes and chemotypes in Fusarium graminearum complex strains isolated from maize fields of northwest Argentina. Int. J. Food Microbiol. 2012, 153, 229-233. [CrossRef] [PubMed]

27. Leslie, J.; Summerell, B. The Fusarium Laboratory Manual; Blackwell Publishing Ltd.: Oxford, UK, 2006.

28. Van der Lee, T.; Zhang, H.; van Diepeningen, A.; Waalwijk, C. Biogeography of Fusarium graminearum species complex and chemotypes: A review. Food Addit. Contam. 2015, 32, 453-463. [CrossRef] [PubMed]

29. Geiser, D.M.; del Mar Jiménez-Gasco, M.; Kang, S.; Makalowska, I.; Veeraraghavan, N.; Ward, T.J.; Zhang, N.; Kuldau, A.G.; O’Donnell, K. FUSARIUM-ID v. 1.0: A DNA sequence database for identifying Fusarium. Eur. J. Plant Pathol. 2004, 110, 473-479. [CrossRef]

30. Fusarium MLST Database. Available online: http:/ /www.westerdijkinstitute.nl/fusarium/ (accessed on 7 March 2018).

(C) 2018 by the authors. Licensee MDPI, Basel, Switzerland. This article is an open access article distributed under the terms and conditions of the Creative Commons Attribution (CC BY) license (http://creativecommons.org/licenses/by/4.0/). 